\title{
Observing the migration of hydrogen species in hybrid perovskite materials through $\mathrm{D} / \mathrm{H}$ isotope exchange
}

\author{
Subha Sadhu, ${ }^{\ddagger}$ Thierry Buffeteau, ${ }^{\dagger}$ Simon Sandrez, ${ }^{\ddagger}$ Lionel Hirsch, ${ }^{\ddagger *}$ Dario M. Bassani ${ }^{\dagger *}$ \\ ${ }^{\sharp}$ Univ. Bordeaux, CNRS, Bordeaux INP, IMS, UMR 5218, ENSCBP F-33405, Talence, France \\ ${ }^{\dagger}$ Univ. Bordeaux, CNRS, Bordeaux INP, ISM, UMR 5255, F-33405, Talence, France
}

\begin{abstract}
Unlike heavier elements, the migration of hydrogen species in perovskite materials cannot be directly tracked using imaging or mass spectrocopy techniques. Our results show that quantitative analysis of D / $\mathrm{H}$ exchange in $\mathrm{PbCH}_{3} \mathrm{ND}_{3} \mathrm{I}_{3}$ allows indirect monitoring of $\mathrm{H}$ migration by following the $\mathrm{N}-\mathrm{D}$ vibration using polarization-modulated infrared reflexion-absorption spectroscopy. Kinetic analysis shows that the isotope exchange process is pseudo-first order and particularly sensitive to the intensity of light and relative humidity, and, to a lesser degree, sample thickness. In the presence of light (450 nm), the D / $\mathrm{H}$ exchange is accelerated up to 10-fold with respect to samples in the dark, but slows again for higher light intensities. This unexpected behavior is in agreement with the Bourgoin-Corbett mechanism for ionization-enhanced ion migration. The technique also allows the direct assessment of the efficiency of protective layers towards deterioration of hybrid organic-inorganic perovskite devices by moisture. Comparison of different monolayer-forming fluorinated molecules reveals important differences in rates attesting to variations between their efficiency in blocking access to the active layer by water molecules.
\end{abstract}

\section{INTRODUCTION}

Hybrid organic inorganic perovskite (HOIP) solar cells have enjoyed tremendous progress over the last decade both in terms of performance and reliability. In these devices, the microcrystalline nature of the active layer is determined by the deposition process and plays an important role in determining the performance by defining parameters such as grain size, ${ }^{1}$ grain boundaries, ${ }^{2}$ density of pinholes, ${ }^{3}$ crystal quality and orientation of the crystalline growth. ${ }^{4}$ Modification of deposition protocols for the active layer have considerably improved issues such as hysteresis, ${ }^{5}$ and there has been strong effort in developing new interfacial materials to reduce recombination ${ }^{6}$ and increase the durability of the devices. ${ }^{7-10}$ The latter remains, however, well below a target of 10 years operational lifetime and represents a major obstacle to the widespread deployment of this technology. Better stability can be obtained using all-inorganic perovskites ${ }^{11}$ or mixed metal cation hybrid perovskites, ${ }^{12}$ but their conversion efficiency is inferior to that of materials incorporating organic cations such as methylammonium, formamidinium, or guanidinium mixed halides. In the latter, ion migration and damage by moisture have been identified as the principal processes leading to the decline of performance during irradiation. ${ }^{13-15}$ Ion migration is accelerated by light, and can be intrinsic to the chemical structure of the material or due to the presence of defects. Studies on deuterium-labelled methylammonium lead iodide perovskites (MAPI) showed that protons at the interface are also labile and can shuttle between vicinal sites through tunneling. 16

Moisture-induced degradation is especially damaging in perovskite devices. Increasing their stability through encapsulation combined with the use of a strongly hydrophobic molecular adlayer has been particularly promising in extending device lifetimes. Along these lines, some groups have shown that a molecular layer of fluorohalogenated compounds could reduce device degradation by moisture. ${ }^{17,18}$ The monolayer, formed through halogen bonding to the MAPI surface, is hydrophobic and therefore constitutes an added barrier for moisture penetrating past the encapsulation and into the active layer. Lifetimes in excess of 100 days were achieved by Ahmad and co-workers using a perfluoroalkyl-substituted imidazolium derivative in lieu of the iodopentafluorophenol. ${ }^{19}$ In this case, the strongly hydrophobic nature of the dopant contributes to the formation of a protective layer which acts as a barrier towards moisture while passivating surface defects. With continued progress in the efficiency of moisture-resistant barriers, ${ }^{20}$ the development of faster and more direct tests of their efficiency becomes crucial to allow fast and efficient screening of multiple strategies as compared to the thousands of hours of continuous irradiation required today.

The overall diffusion of any ion into the microcrystalline active layer is proposed to occur as a combination of ion migration at grain boundaries and in the bulk of grains. ${ }^{21,22}$ The interplay between ion migration and stability is also evidenced in studies on 2D / 3D perovskites, where reduced ion diffusion favorably impacts device lifetime. ${ }^{23}$ Ion migration negatively impacts the development of protection strategies because device lifetime intrinsically depends on the active layer morphology, grain boundaries, ${ }^{24}$ and more generally on the device architecture as a whole. ${ }^{20}$ For this reason, studies on device lifetime are documented using a large number of devices to obtain statistically meaningful results. Obviously, experimental approaches relying on monitoring water uptake would be much more convenient. Unfortunately, techniques such as TOF-SIMS, which have previously been used to evidence migration of oxygen, ${ }^{25,26}$ 
alkyl ammoniums ${ }^{27}$ or iodide, ${ }^{28}$ or transient capacitance measurements, ${ }^{29}$ are not readily applicable to hydrogen species $\left(\mathrm{H}^{+}, \mathrm{H}^{0}, \mathrm{H}^{-1}\right)$ due to hydrogen's small size and mass. The latter is instead well suited for neutron scattering experiments, ${ }^{30}$ but such studies require access to large-scale radiation facilities. The paucity of experimental techniques for tracking the migration of hydrogen species has been noted and contrasts the significance of understanding its contribution to the stability of HOIP devices. ${ }^{31}$ Migration of intrinsic protons in HOIP materials has also been linked to intrinsic doping. ${ }^{32}$

During the course of our studies on interfacial effects on hybrid perovskite devices, we recently reported the use of polarization-modulated infrared reflection-absorption spectroscopy (PM-IRRAS) to follow and characterize monolayer formation on MAPI surfaces. ${ }^{33}$ This spectroscopic technique exploits the IRRAS advantages of the electric field enhancement and the surface selection rules and is widely used to characterize self-assembled monolayers grafted onto gold surfaces. ${ }^{34-37}$ The observed IR transitions can be readily correlated to the different functional groups of the molecules present on the surface, much the same way as IR spectroscopy

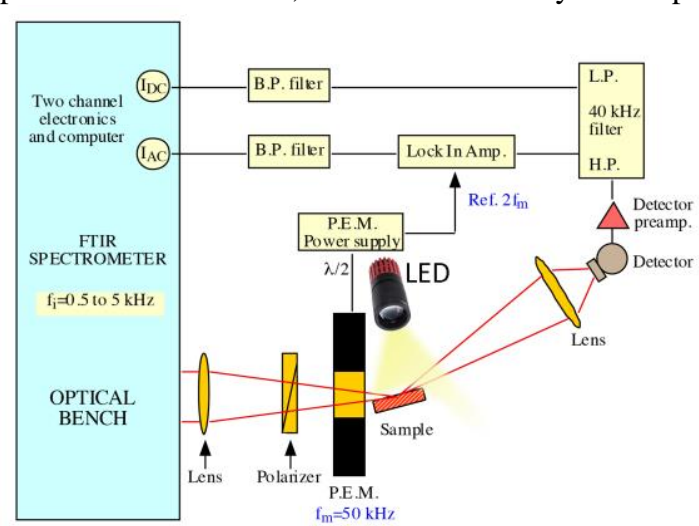

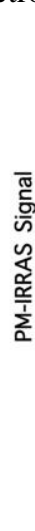

can be used to identify molecules in solution or in the solid. More importantly, the intensity of the PM-IRRAS bands is proportional to the quantity of molecules on the surface as well as to their orientation. We now report that this technique can also be used to indirectly follow the migration of hydrogen species in HOIP materials by exploiting the difference in vibrational frequency of $\mathrm{X}-\mathrm{H}$ vs. $\mathrm{X}-\mathrm{D}$ bonds, where $\mathrm{X}$ is a heavier element such as carbon, oxygen, or nitrogen. Our results show that this technique is very powerful and allows direct probing of environmental effects, such as relative humidity and the effect of light, on the migration of hydrogen species. The latter is particularly interesting as it shows that effect of light on the rate of hydrogen species migration is non-monotonic, being very slow in the absence of light, highest at low to medium light levels, and then decreasing at higher irradiation intensities. This technique also allows the indirect assessment of the protection against moisture offered by surface modification of the active layer by simply monitoring the rate of hydrogen exchange within the active layer in the presence of ambient moisture as shown in Figure 1 .
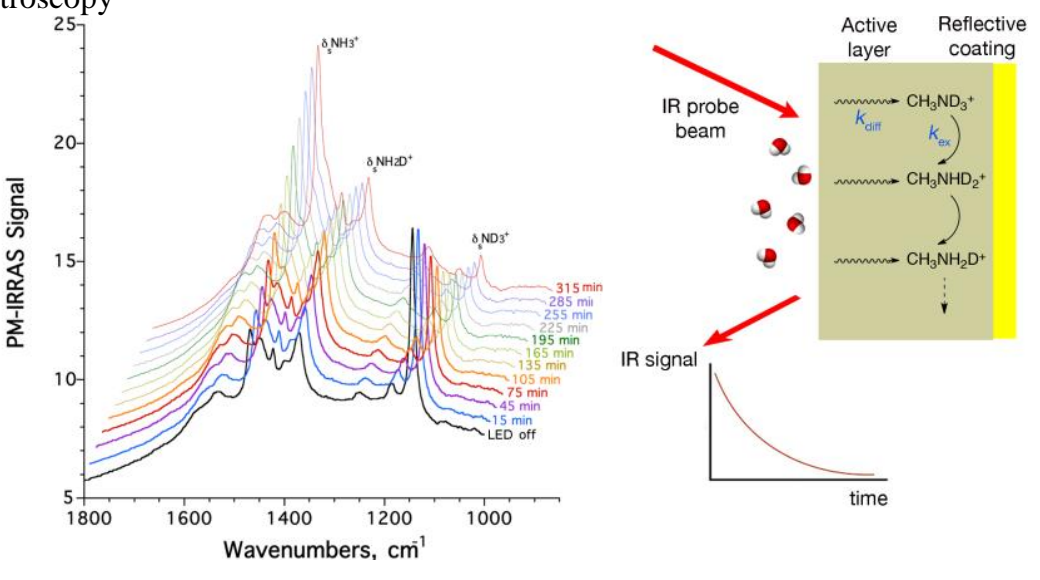

Figure 1. Experimental set-up for PM-IRRAS incorporating a variable power LED light source (left) and typical spectra of the region of interest $\left(800-1800 \mathrm{~cm}^{-1}\right)$ obtained at different time intervals following illumination of the sample (center). The bands assigned to the $\delta_{\mathrm{s}}$ vibrations of the $\mathrm{ND}_{3}{ }^{+}, \mathrm{NH}_{2} \mathrm{D}^{+}$, and $\mathrm{NH}_{3}{ }^{+}$species are shown (see SI for complete spectral assignment). The overall changes occurring in the material during the acquisition are illustrated (right panel) to highlight the contribution of diffusion and isotope exchange to the variations in the PM-IRRAS signal.

\section{RESULTS AND DISCUSSION}

Methylammonium lead iodide perovskites contain labile protons located on the ammonium fragment that contribute to ion migration. To evidence hydrogen migration, we proceeded to investigate the $\mathrm{D} / \mathrm{H}$ exchange occurring in MAPI films prepared using deuterated MAI $\left(\mathrm{CH}_{3} \mathrm{ND}_{3} \mathrm{I}\right)$. The latter is easily prepared by repeated proton / deuterium exchange in deuterium oxide followed by drying under vacuum. Films (80 $-600 \mathrm{~nm}$ thick) of MAPI on gold-coated glass substrates were prepared as described previously. ${ }^{33}$ Gold substrates are used as a reflective interface for the PM-IRRAS experiments because this allows a double absorption in the thin films. The PMIRRAS spectra from the deuterated MAPI exhibit broad intense bands at $3500-3000$ and $2500-2000 \mathrm{~cm}^{-1}$, assigned to the $\mathrm{N}-\mathrm{H}$ and $\mathrm{N}-\mathrm{D}$ stretching vibrational modes, respectively. The region at lower wavenumbers, between 1800 and $1000 \mathrm{~cm}^{-1}$, contains the symmetric and asymmetric bending ( $\delta_{\mathrm{s}}$ and $\delta_{\mathrm{a}}$, respectively) as well as rocking (r) vibrations of the $\mathrm{N}-\mathrm{D}$ and $\mathrm{N}-\mathrm{H}$ species. Although less intense, the bands related to the bending modes are sharper and contain more information than the stretching modes at higher wavenumbers and were therefore chosen to follow the $\mathrm{N}-\mathrm{H}$ vs. N-D composition of the material over time.

\section{Kinetic analysis of PM-IRRAS experiments and spectral assignment}

The PM-IRRAS experiments were conducted in a controlled dry environment protected from ambient light $(296 \mathrm{~K}, \mathrm{RH}=$ $3 \%$ ) using the setup shown in Figure 1. A 450-nm LED source was used to irradiate the sample during the experiments and a calibrated powermeter was used to measure the light intensity at the sample location. Spectra acquired at different time intervals show a gradual evolution of the sample that is characterized by a decrease in the intensity of the band at 1144 $\mathrm{cm}^{-1}$ assigned to the $\delta_{\mathrm{s}} \mathrm{ND}_{3}{ }^{+}$vibrational mode. This indicates that the $\mathrm{CH}_{3} \mathrm{ND}_{3}{ }^{+}$species is undergoing a chemical transformation resulting in its disappearance. Concomitantly, we observe the appearance of a signal at $1469 \mathrm{~cm}^{-1}$ corresponding to the formation of the $\mathrm{CH}_{3} \mathrm{NH}_{3}{ }^{+}$species, ${ }^{38}$ as well as that of an intermediate $\mathrm{CH}_{3} \mathrm{NH}_{2} \mathrm{D}^{+}$species (at 1369 $\mathrm{cm}^{-1}$ ) whose intensity first increases, and then decreases (see spectra in Figure 1). In solid-state transformations, the 
concentrations of reactants are not generally used. The formation or disappearance of a species is instead described by the conversion fraction $(\alpha)$, defined here as the molar fraction of each MA species present (eq. 1):

$$
\alpha(t)=\frac{I_{0}-I_{t}}{I_{0}-I_{\infty}}
$$

, where $I_{t}, I_{0}$ and $I_{\mathrm{t}}$ are the intensity of the PM-IRRAS signal of a specific MA species $\left(\mathrm{CH}_{3} \mathrm{ND}_{3}{ }^{+}, \mathrm{CH}_{3} \mathrm{NHD}_{2}{ }^{+}, \mathrm{CH}_{3} \mathrm{NH}_{2} \mathrm{D}^{+}\right.$, or $\mathrm{CH}_{3} \mathrm{NH}_{3}{ }^{+}$) at a time $t, t=0$, or $t=\infty$, respectively. Conversion factors, like concentrations, can be plotted as a function of independent variables to provide mechanistic information. ${ }^{39}$

We rapidly remarked that the evolution of the material, as shown by the relative intensity of the $\delta_{\mathrm{S}} \mathrm{ND}_{3}{ }^{+}$vibration, depends on the amount of light received by the sample. The effect of light on the loss of the $\delta_{\mathrm{S}} \mathrm{ND}_{3}{ }^{+}$signal is shown in Figure $2 \mathrm{~A}$, and it is immediately apparent that the deuterium exchange process is significantly accelerated by irradiation. The effect is stopped once the light is switched off and reactivated when the light is turned on again. Figure 2A shows multiple on / off cycles with similar rates during the dark and irradiated periods. At light irradiance of $0.5 \mathrm{~mW} / \mathrm{cm}^{2}$ (corresponding to $1.1 \times 10^{15}$ photons $\mathrm{s}^{-1} \mathrm{~cm}^{-2}$ ), we find that a $375-\mathrm{nm}$ thick sample undergoes ca. $85 \%$ loss of the $\delta_{\mathrm{S}} \mathrm{ND}_{3}{ }^{+}$ signal over a period of $5 \mathrm{~h}$ (Figure 2B). During this time, the vibrational signal corresponding to the $\mathrm{CH}_{3} \mathrm{ND}_{3}{ }^{+}$species decreases, whereas those corresponding to the intermediate $\mathrm{CH}_{3} \mathrm{NH}_{2} \mathrm{D}^{+}$species first increase, and then decrease in intensity, and those corresponding to the formation of the $\mathrm{CH}_{3} \mathrm{NH}_{3}{ }^{+}$species grow in. The behavior is fully consistent with a mechanism in which the deuterium atoms in MAI undergo sequential substitution in a step-wise mechanism, as shown in Figure 2. In agreement with this, the appearance of the $\delta_{\mathrm{s}} \mathrm{NH}_{3}{ }^{+}$signal is slower than that for the loss of $\delta_{\mathrm{S}} \mathrm{ND}_{3}{ }^{+}$ signal, and presents an induction period ascribed to the formation of the intermediate $\mathrm{CH}_{3} \mathrm{NHD}_{2}{ }^{+}$and $\mathrm{CH}_{3} \mathrm{NH}_{2} \mathrm{D}^{+}$ species. Interestingly, only the latter species, clearly identified by its signal at $1369 \mathrm{~cm}^{-1}$, grows in and disappears, whereas those assigned to the $\mathrm{CH}_{3} \mathrm{NHD}_{2}^{+}$intermediate (at $1185 \mathrm{~cm}^{-1}$ ) remain small throughout the exchange process. This suggests that the latter is consumed at a rate that is similar to or faster than its formation, and that it does not accumulate in significant amounts during the course of the experiment. ${ }^{40}$
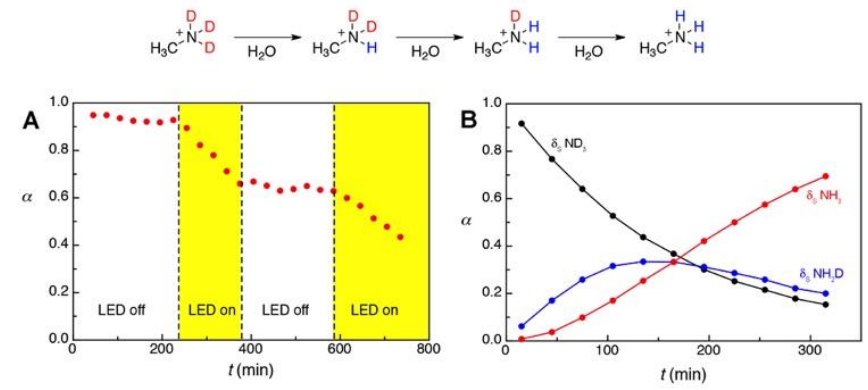

Figure 2. A: Conversion factor $(\alpha)$ for the $\mathrm{CH}_{3} \mathrm{ND}_{3}{ }^{+}$species followed over time on a sample undergoing on / off cycles of irradiation (450 nm, $0.5 \mathrm{~mW} \mathrm{~cm} \mathrm{~cm}^{-2}, 80-\mathrm{nm}$ thick sample). B: Conversion fraction $(\alpha)$ determined from signals assigned to $\delta_{\mathrm{s}} \mathrm{NH}_{3}{ }^{+}, \delta_{\mathrm{S}} \mathrm{NH}_{2} \mathrm{D}^{+}$, and $\delta_{\mathrm{S}} \mathrm{ND}_{3}{ }^{+}$for a $375-\mathrm{nm}$ thick sample exposed to $3 \% \mathrm{RH}(296 \mathrm{~K})$ and $450 \mathrm{~nm}$ light $\left(0.5 \mathrm{~mW} \mathrm{~cm}^{-2}\right)$, lines are guides for the eyes. The behavior is consistent with a mechanism in which the deuterium atoms in $d_{3}$-MAI undergo sequential substitution in a step-wise mechanism as shown in the scheme above.

\section{Assignment of the rate-limiting process}

The data for the loss of $\mathrm{CH}_{3} \mathrm{ND}_{3}{ }^{+}$species as shown in Figure $2 \mathrm{~B}$ is in overall agreement with a reaction model governed by either diffusion of the reacting species or by the rate of exchange at each reaction site. Alternative solid-state reaction models, such as geometrical contraction or nucleation, instead exhibit either power law or sigmoidal evolution of the conversion fraction as a function of time, respectively, that deviate significantly from our experimental observations. ${ }^{39,41}$ To investigate this further, the loss of the $\delta_{\mathrm{s}} \mathrm{ND}_{3}{ }^{+}$signal in an 80 -nm thick sample upon exposure to moisture and light (3\% $\mathrm{RH}$ at $296 \mathrm{~K}, 1 \mathrm{~mW} / \mathrm{cm}^{2}$ at $450 \mathrm{~nm}$ ) was followed over a period of 10 hours. The result is shown in Figure 3A and we find that the data fits well for a first-order reaction-limited model with a pseudo first-order rate constant $k=1.2 \pm 0.1 \mathrm{x}$ $10^{-4} \mathrm{~s}^{-1}$, whereas fitting according to 2-D or 3-D diffusion models is not satisfactory (see SI). In comparison, the rate of exchange of $\mathrm{H}^{+}$in methylammonium ( $k_{\mathrm{ex}}$, Fig.1) was determined to be $4.4 \times 10^{8} \mathrm{M}^{-1} \mathrm{~s}^{-1}$, , which is several orders of magnitude faster than the process observed here. From this, we conclude that the exchange process is rate-limited by the rate of diffusion ( $k_{\text {diff }}$, Fig. 1$)$, which could itself be limited by the rate of generation or trapping of the migrating species.
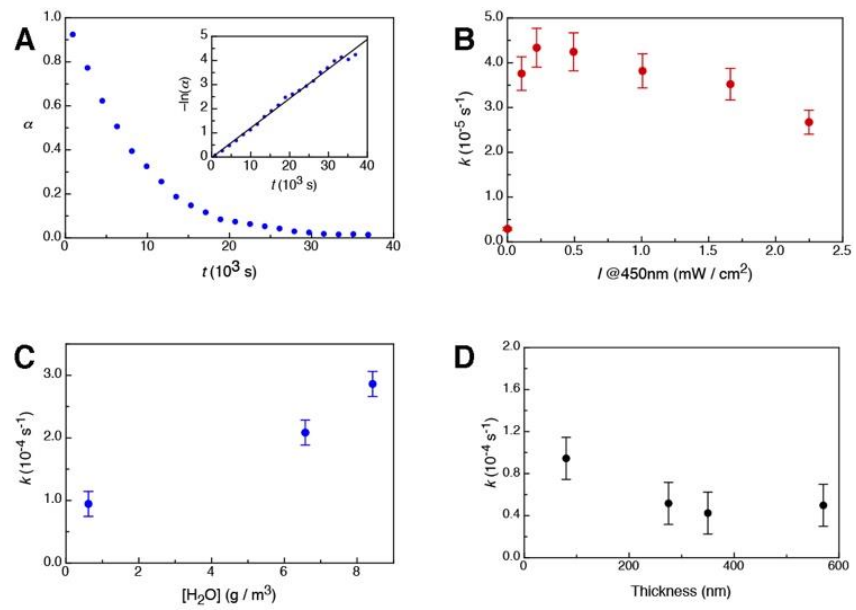

Figure 3. Effect of external parameters (light, relative humidity, sample thickness) on the rate of deuterium exchange (as determined from the conversion factor $\alpha$ for the loss of the $\delta_{\mathrm{S}} \mathrm{ND}_{3}{ }^{+}$signal). A: Conversion factor $\alpha$ for the loss of the $\delta_{\mathrm{S}} \mathrm{ND}_{3}$ signal in an $80-\mathrm{nm}$ thick sample $\left(450 \mathrm{~nm}, 1 \mathrm{~mW} \mathrm{~cm}^{-2}\right)$ plotted in linear and semi-logarithmic (inset) axes. B: Dependence of exchange rate $(k)$ on the irradiation intensity (at $450 \mathrm{~nm}$ ) for a $350-\mathrm{nm}$ thick sample. C: Calculated rates vs. ambient water concentration for $80-\mathrm{nm}$ thick samples $\left(450 \mathrm{~nm}, 0.5 \mathrm{~mW} \mathrm{~cm}{ }^{-2}\right)$. D. Calculated rates of $\mathrm{D} / \mathrm{H}$ exchange plotted for samples of different thicknesses $\left(450 \mathrm{~nm}, 0.5 \mathrm{~mW} \mathrm{~cm}^{-2}\right.$ ).

Effect of light intensity, water concentration, and thickness.

As already mentioned, the rate of the exchange process is sensitive to the intensity of the incident light. A plot of the calculated rate vs. irradiation intensity for a 350-nm thick sample is shown in Figure 3B. The observed rate for the loss of $\mathrm{CH}_{3} \mathrm{ND}_{3}{ }^{+}$species increases rapidly at low light intensities to reach a maximum at $0.25 \mathrm{~mW} \mathrm{~cm}^{-2}\left(5.5 \times 10^{14}\right.$ photons s $\mathrm{cm}^{-1}$ ${ }^{2}$ ), and then decreases at larger irradiances. ${ }^{43}$ In the absence of 
light, the exchange process is very slow, with $k_{\text {dark }}=3.0 \pm 0.6$ $\mathrm{x} 10^{-6} \mathrm{~s}^{-1}$. Light-induced acceleration of ion migration has been previously documented for iodine ${ }^{44,45}$ and methylammonium, ${ }^{46}$ as well as for the interstitial diffusion of molecular oxygen. ${ }^{25,26}$ However, the effect of light is generally observed to tend towards a maximum value rather than to decrease at higher light dosage as observed here. Such behavior is indicative of competing processes at low and high light intensities and sheds light on the migration mechanism responsible for the deuterium exchange in the perovskite material. Based on absorptivity data for HOIP calculated from photothermal deflection spectroscopy, we expect that $>97 \%$ of the incident light is absorbed by samples $\geq 80 \mathrm{~nm}$ thick at $450 \mathrm{~nm}^{47}$ In agreement with the intervention of ambient moisture in the rate-limiting step, we find that the rate of deuterium exchange is proportional to the concentration of water vapor to which the sample is exposed. The latter effect is shown in Figure 3C, giving a roughly three-fold increase in the exchange rate upon increasing the relative humidity from $3 \%$ to $41 \%$ (at $296 \mathrm{~K}$ ). The effect of sample thickness is shown in Figure 3D for four samples $(80-570 \mathrm{~nm})$, and indicates that the exchange is only weakly dependent on the sample thickness except for very thin samples $(80 \mathrm{~nm})$, where interfacial trapping of charge carriers may become important.

\section{Mechanism of isotope exchange}

From the experimental observations, a possible model for the D / $\mathrm{H}$ exchange process emerges. For complete exchange to take place, either protons or water must diffuse throughout the material. Significant penetration of water seems unlikely since we do not observe signals attributable to $\mathrm{H}_{2} \mathrm{O}$ or $\mathrm{D}_{2} \mathrm{O}$ in the PMIRRAS spectra, even for samples exposed to $32 \%$ $\mathrm{RH}^{48}$ If we assume that the exchange is limited by proton diffusion in the bulk, then application of a 1D diffusion model yields diffusion constants that are small and dependent on sample thickness (e.g. $1.5 \pm 0.3 \times 10^{-15} \mathrm{~cm}^{2} \mathrm{~s}^{-1}$ for a $350-\mathrm{nm}$ thick sample in the dark at $\mathrm{RH}=3 \%) .{ }^{49}$ This value is comparable to that observed for the migration of $\mathrm{O}_{2}$, a small diatomic molecule, in MAPI at room temperature. ${ }^{26}$

An alternative explanation is that water undergoes partial dissociation at the MAPI surface and that his, or the initial diffusion of protons, may be rate-limiting. In their theoretical investigation of the structure and energetics of the migration of hydrogenic impurities in MAPI, Egger, Kronic and Rappe propose that ionization-enhanced hydrogen migration occurs in hybrid perovskites. ${ }^{50,51}$ The process involves the capture of electrons by $\mathrm{H}^{+}$, which is transformed into $\mathrm{H}^{*}$. The new species then relaxes by moving to an adjacent lower energy site, where it may again undergo electron or hole capture and migrate to a different site to minimize the total energy. At each location where $\mathrm{H}^{+}$is generated, $\mathrm{H}^{+} / \mathrm{D}^{+}$exchange may occur and result in a change of the PM-IRRAS signals of the $\mathrm{N}-\mathrm{D}$ bonds (Figure 4).

In this mechanism, we expect that the rate of $\mathrm{H}^{+} / \mathrm{D}^{+}$ exchange will increase with increasing light intensity, but not with sample thickness provided that all of the incident light is absorbed. This is indeed what we observe for light intensities $\leq 0.5 \mathrm{~mW} \mathrm{~cm}^{-2}$ as shown in Figures 3B (light intensity) and $3 \mathrm{D}$ (thickness). At higher light intensities, the $\mathrm{H}^{+} / \mathrm{D}^{+}$ exchange rate then decreases in agreement with the presence of a competing process that retards migration. We speculate that the latter process may be the result of recombination between radical $\left(\mathrm{H}^{*}\right.$ and $\left.\mathrm{I}^{*}\right)$ or ionic species $\left(\mathrm{H}^{+}\right.$and $\mathrm{H}^{-}$or $\left.\mathrm{I}^{-}\right)$, whose overall concentration would also increase with increasing light intensity, to generate $\mathrm{H}_{2}$ and $\mathrm{HI}$. Products that would result from recombination (including $\mathrm{H}_{2}$ and $\mathrm{I}_{2}$ ) have been previously identified by mass spectrometry and their presence correlated to the degradation of the active layer. ${ }^{52}$

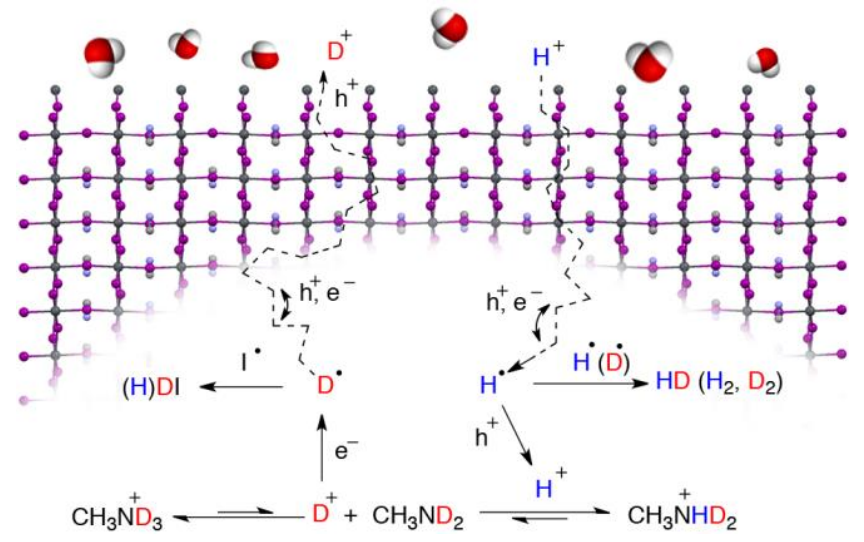

Figure 4. Possible mechanism for D / H exchange in HOIP during illumination based on ionization-enhanced ion migration in which repetitive oxidation and reduction steps accelerate diffusion of charged species (only reduction is shown for clarity). ${ }^{50,51}$ Coupling between radical or charged species induces trapping of the migrating species and may account for the slower isotope exchange observed at higher light irradiance.

\section{Application to the evaluation of protective adlayers}

Beyond contributing to the understanding of the fundamental processes governing proton migration in HOIPs, these results reveal a new approach to rapidly screen new materials for abating damage caused by water or moisture. As pointed out above, current methodologies for assessing the performance of new moisture barriers rely on the statistical analysis of device operational lifetimes and their comparison with control devices, which is time-consuming and dependent on the device architecture. Instead, our results show that PMIRRAS, and more generally IR spectroscopy, can provide indirect measurement of the amount of moisture in contact with the active layer. The preparation of the deuterated MAI material is straightforward and inexpensive, and the results are not highly dependent on the device thickness. To test this, we proceeded to compare the rates of $\mathrm{H} / \mathrm{D}$ exchange for MAPI films treated with a monolayer coverage of various fluorinated molecules chosen for their hydrophobicity: perfluorooctane (PFO), 1H,1H,2H,2H-perfluorodecanethiol (PFDT), iodopentafluorobenzene (IPFB), and $N$-(5,5,5,4,4,3,3heptafluoro- $n$-pentyl)- $N$ '-methylimidazolium iodide (FIM). Fluorinated hydrocarbons are highly hydrophobic and considered to be amongst the most promising candidates for the formation of water barriers in HOIP devices. We previously showed that PFO spontaneously forms sparse films on the MAPI surface, whereas PFDT forms much more dense films in which the fluoroalkyl chains lie flat on the surface due to fluorine - perovskite interactions. ${ }^{33}$ IPFB was previously reported by Snaith and co-workers to form dense monolayers on MAPI surface as a result of halogen bonding. ${ }^{17}$ The use of FIM for the protection of HOIP devices was reported by Ahmad and co-workers to yield devices that could resist exposition to ambient moisture in excess of 100 days without loss of performance. ${ }^{19}$ 
The effect of surface modification using the different fluorocarbons on the rate of $\mathrm{H} / \mathrm{D}$ exchange is presented in Figure 5 . The protective layers were deposited by exposing the perovskite surface to the neat fluorocarbon $(15 \mathrm{~min})$ and rinsing the excess using $\alpha, \alpha, \alpha$,-trifluorotoluene to afford a single or few-molecule layer on the surface. ${ }^{17,33}$ In the case of FIM, the fluorocarbon was added to the precursor solution prior to deposition of the active layer (see SI for details). ${ }^{19}$ In this experiment, the samples were exposed to an environment of $3 \% \mathrm{RH}(296 \mathrm{~K})$ and illumination $\left(450 \mathrm{~nm}, 0.5 \mathrm{~mW} \mathrm{~cm}{ }^{-2}\right)$ while following the loss of the $\delta_{\mathrm{S}} \mathrm{ND}_{3}{ }^{+}$signal (see SI for details). We find that both the PFDT and IPFB monolayers considerably retard the exchange process, whereas PFO offers only partial protection with respect to the control. This is in line with the greater hydrophobicity of the more compact PFDT and IPFB monolayers compared to PFO. Additionally, the effect of IPFB is greater than that of PFDT, which may also reflect the different molecular surface packing of the two molecules. In agreement with the greater protection reported for the FIM compound, ${ }^{19}$ we find that active layers incorporating this passivation method provide the slowest rate constant for H / D exchange. While the present work does not establish a direct link between a protective layer's efficiency towards slowing the diffusion of hydrogen species with its ability to protect a device towards damage by moisture, both $\mathrm{H}$ / D exchange and damage by moisture are caused by contact with water molecules. Therefore, protective adlayers that reduce the access to the HOIP layer by water molecules are also expected to slow $\mathrm{H} / \mathrm{D}$ exchange.

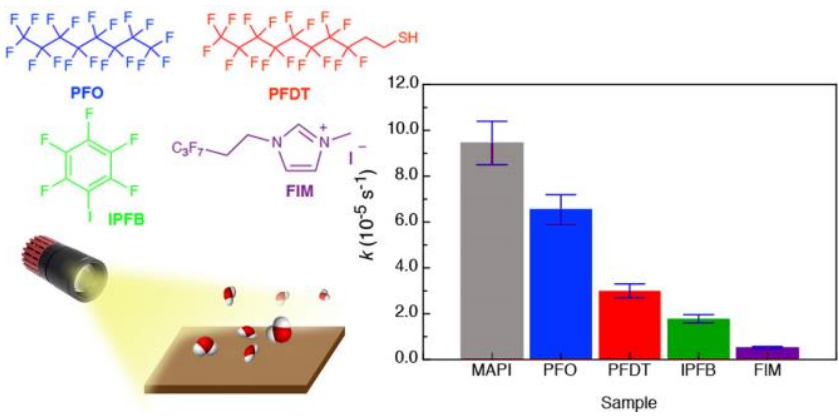

Figure 5. Effect of surface protection afforded by adlayers of fluorinated molecules as evaluated by the rate of $\mathrm{H} / \mathrm{D}$ exchange in MAPI samples exposed to $3 \% \mathrm{RH}(296 \mathrm{~K})$ and illumination (450 nm, $0.5 \mathrm{~mW} \mathrm{~cm}^{-2}$ ).

\section{CONCLUSIONS}

Understanding ion migration is an important step in improving HOIP-based devices. Although migration of heavy ions such as $\mathrm{I}^{-}$and $\mathrm{MAI}^{+}$has been investigated using various techniques, these same techniques are not directly applicable to following migration of hydrogen species. We have shown that relatively straightforward IR spectroscopic techniques allow, when coupled to isotopic labeling, to follow the migration of hydrogen species inside the active layer and that this can be used to elucidate the effect of the operational environment (light, ambient humidity) on the rate of migration. The effect of light appears to be in agreement with the ionization-activated mechanism for the migration of hydrogen species as proposed by Egger et al. for HOIP materials. ${ }^{50}$ The overall rate of $\mathrm{H} / \mathrm{D}$ exchange can be modeled using first-order kinetics and this provides a numerical handle that can be used to directly probe the effectiveness of surface modification on water accessibility. Using vibrational spectroscopy combined with isotopic labeling thus provides a tool to directly measure water access to the HOIP active layer that can be used to quickly screen potential molecular barriers to moisture. More conventional IR techniques, such as transmission FTIR, could be used to perform similar experiments on perdeuterated MAPI samples spin-coated onto IR-transparent substrates such as $\mathrm{CaF}_{2}$.

\section{ASSOCIATED CONTENT}

\section{Supporting Information}

Supplementary Information (SI) available: details of film preparation and PM-IRRAS experiments, evaluation of different reaction models, analysis of protection efficiency of protective layers (PDF).

The Supporting Information is available free of charge on the ACS Publications website.

\section{AUTHOR INFORMATION}

\section{Corresponding Author}

*lionel.hirsch@ims-bordeaux.fr; dario.bassani@u-bordeaux.fr

\section{Author Contributions}

The manuscript was written through contributions of all authors.

\section{ACKNOWLEDGMENT}

The authors are grateful for financial support from the ANR under grants LabEx AMADEus (ANR-10-LABX-0042-AMADEUS through grant ANR-10-IDEX-0003-02) and ANR grant ANR-18CE05-0021-01. The Aquitaine government is thanked for support through grant 2019-1R1M08.

\section{REFERENCES}

(1) Nie, W.; Tsai, H.; Asadpour, R.; Blancon, J.-C.; Neukirch, A. J.; Gupta, G.; Crochet, J. J.; Chhowalla, M.; Tretiak, S.; Alam, M. A.; Wang, H.-L.; Mohite, A. D. High-efficiency solution-processed perovskite solar cells with millimeter-scale grains. Science 2015, 347, $522-525$.

(2) Wen, X.; Sheng, R.; Ho-Baillie, A. W. Y.; Benda, A.; Woo, S.; Ma, Q.; Huang, S.; Green, M. A. Morphology and Carrier Extraction Study of Organic-Inorganic Metal Halide Perovskite by One- and Two-Photon Fluorescence Microscopy. J. Phys. Chem. Lett. 2014, 5, 3849-3853.

(3) Huang, Z.; Wang, D.; Wang, S.; Zhang, T. Highly Efficient and Stable $\mathrm{MAPbI}_{3}$ Perovskite Solar Cell Induced by Regulated Nucleation and Ostwald Recrystallization. Materials 2018, 11, 778778

(4) Passarelli, J. V.; Fairfield, D. J.; Sather, N. A.; Hendricks, M. P.; Sai, H.; Stern, C. L.; Stupp, S. I. Enhanced Out-of-Plane Conductivity and Photovoltaic Performance in $\mathrm{n}=1$ Layered Perovskites through Organic Cation Design. J. Am. Chem. Soc. 2018, 140, 7313-7323.

(5) Ahn, N.; Son, D.-Y.; Jang, I.-H.; Kang, S. M.; Choi, M.; Park, N.-G. Highly Reproducible Perovskite Solar Cells with Average Efficiency of $18.3 \%$ and Best Efficiency of $19.7 \%$ Fabricated via Lewis Base Adduct of Lead(II) Iodide. J. Am. Chem. Soc. 2015, 137, 8696-8699.

(6) Zhang, F.; Shi, W.; Luo, J.; Pellet, N.; Yi, C.; Li, X.; Zhao, X.; Dennis, T. J. S.; Li, X.; Wang, S.; Xiao, Y.; Zakeeruddin, S. M.; Bi, D.; Grätzel, M. Isomer-Pure Bis-PCBM-Assisted Crystal Engineering of Perovskite Solar Cells Showing Excellent Efficiency and Stability. Adv. Mat. 2017, 29, 1606806-1606806.

(7) Yang, S.; Wang, Y.; Liu, P.; Cheng, Y.-B.; Zhao, H. J.; Yang, H. G. Functionalization of perovskite thin films with moisturetolerant molecules. Nat. Energy 2016, 1, 15016. 
(8) Hou, Y.; Zhou, Z. R.; Wen, T. Y.; Qiao, H. W.; Lin, Z. Q.; Ge, B.; Yang, H. G. Enhanced moisture stability of metal halide perovskite solar cells based on sulfur-oleylamine surface modification. Nanoscale Horiz. 2019, 4, 208-213.

(9) Wei, D.; Huang, H.; Cui, P.; Ji, J.; Dou, S.; Jia, E.; Sajid, S.; Cui, M.; Chu, L.; Li, Y.; Jiang, B.; Li, M. Moisture-tolerant supermolecule for the stability enhancement of organic-inorganic perovskite solar cells in ambient air. Nanoscale 2019, 11, 1228-1235.

(10) Yang, S.; Chen, S.; Mosconi, E.; Fang, Y.; Xiao, X.; Wang, C.; Zhou, Y.; Yu, Z.; Zhao, J.; Gao, Y.; De Angelis, F.; Huang, J. Stabilizing halide perovskite surfaces for solar cell operation with wide-bandgap lead oxysalts. Science 2019, 365, 473-478.

(11) Zhou, Y.; Zhao, Y. Chemical stability and instability of inorganic halide perovskites. Energy Environ. Sci. 2019, 12, 14951511.

(12) Prasanna, R.; Leijtens, T.; Dunfield, S. P.; Raiford, J. A.; Wolf, E. J.; Swifter, S. A.; Werner, J.; Eperon, G. E.; de Paula, C.; Palmstrom, A. F.; Boyd, C. C.; van Hest, M. F. A. M.; Bent, S. F.; Teeter, G.; Berry, J. J.; McGehee, M. D. Design of low bandgap tinlead halide perovskite solar cells to achieve thermal, atmospheric and operational stability. Nat. Energy, 2019, 4, 939-947.

(13) Huang, J.; Tan, S.; Lund, P. D.; Zhou, H. Impact of H2O on organic-inorganic hybrid perovskite solar cells. Energy Environ. Sci. 2017, 10, 2284-2311.

(14) Lee, H.; Gaiaschi, S.; Chapon, P.; Tondelier, D.; Bourée, J.E.; Bonnassieux, Y.; Derycke, V.; Geffroy, B. Effect of Halide Ion Migration on the Electrical Properties of Methylammonium Lead TriIodide Perovskite Solar Cells. J. Phys. Chem. C 2019, 123, 1772817734

(15) DuBose, J. T.; Kamat, P. V. TiO ${ }_{2}$-Assisted Halide Ion Segregation in Mixed Halide Perovskite Films. J. Am. Chem. Soc. 2020, $142,5362-5370$

(16) Chen, Y. F.; Tsai, Y. T.; Hirsch, L.; Bassani, D. M. Kinetic Isotope Effects Provide Experimental Evidence for Proton Tunneling in Methylammonium Lead Triiodide Perovskites. J. Am. Chem. Soc 2017, 139, 16359-16364.

(17) Abate, A.; Saliba, M.; Hollman, D. J.; Stranks, S. D.; Wojciechowski, K.; Avolio, R.; Grancini, G.; Petrozza, A.; Snaith, H. J. Supramolecular halogen bond passivation of organic-inorganic halide perovskite solar cells. Nano Lett. 2014, 14, 3247-3254.

(18) Wolff, C. M.; Canil, L.; Rehermann, C.; Ngoc Linh, N.; Zu, F.; Ralaiarisoa, M.; Caprioglio, P.; Fiedler, L.; Stolterfoht, M.; Kogikoski, S.; Bald, I.; Koch, N.; Unger, E. L.; Dittrich, T.; Abate, A.; Neher, D. Perfluorinated Self-Assembled Monolayers Enhance the Stability and Efficiency of Inverted Perovskite Solar Cells. ACS Nano 2020, 14, 1445-1456.

(19) Salado, M.; Ramos, F. J.; Manzanares, V. M.; Gao, P.; Nazeeruddin, M. K.; Dyson, P. J.; Ahmad, S. Extending the Lifetime of Perovskite Solar Cells using a Perfluorinated Dopant. ChemSusChem 2016, 9, 2708-2714.

(20) Wang, Q.; Phung, N.; Di Girolamo, D.; Vivo, P.; Abate, A Enhancement in lifespan of halide perovskite solar cells. Energy Environ. Sci. 2019, 12, 865-886.

(21) Yang, B.; Brown, C. C.; Huang, J.; Collins, L.; Sang, X.; Unocic, R. R.; Jesse, S.; Kalinin, S. V.; Belianinov, A.; Jakowski, J.; Geohegan, D. B.; Sumpter, B. G.; Xiao, K.; Ovchinnikova, O. S. Enhancing Ion Migration in Grain Boundaries of Hybrid OrganicInorganic Perovskites by Chlorine. Adv. Funct. Mater. 2017, 27, 1700749.

(22) Walsh, A.; Stranks, S. D. Taking Control of Ion Transport in Halide Perovskite Solar Cells. ACS Energy Lett. 2018, 3, 1983-1990.

(23) Schlipf, J.; Hu, Y.; Pratap, S.; Biessmann, L.; Hohn, N.; Porcar, L.; Bein, T.; Docampo, P.; Mueller-Buschbaum, P. Shedding Light on the Moisture Stability of 3D/2D Hybrid Perovskite Heterojunction Thin Films. ACS Appl. Energy Mater. 2019, 2, 10111018.

(24) Chu, Z.; Yang, M.; Schulz, P.; Wu, D.; Ma, X.; Seifert, E.; Sun, L.; Li, X.; Zhu, K.; Lai, K. Impact of grain boundaries on efficiency and stability of organic-inorganic trihalide perovskites. Nat. Commun. 2017, 8, 1-8.
(25) Navickas, E.; Chen, Y.; Lu, Q.; Wallisch, W.; Huber, T. M.; Bernardi, J.; Stöger-Pollach, M.; Friedbacher, G.; Hutter, H.; Yildiz, B.; Fleig, J. Dislocations Accelerate Oxygen Ion Diffusion in $\mathrm{La}_{0.8} \mathrm{Sr}_{0.2} \mathrm{MnO}_{3}$ Epitaxial Thin Films. ACS Nano 2017, 11, 1147511487.

(26) Senocrate, A.; Acartuerk, T.; Kim, G. Y.; Merkle, R.; Starke, U.; Graetzel, M.; Maier, J. Interaction of oxygen with halide perovskites. J. Mater. Chem. A 2018, 6, 10847-10855.

(27) Harvey, S. P.; Li, Z.; Christians, J. A.; Zhu, K.; Luther, J. M.; Berry, J. J. Probing Perovskite Inhomogeneity beyond the Surface: TOF-SIMS Analysis of Halide Perovskite Photovoltaic Devices. ACS App. Mat. Interf. 2018, 10, 28541-28552.

(28) deQuilettes, D. W.; Zhang, W.; Burlakov, V. M.; Graham, D. J.; Leijtens, T.; Osherov, A.; Bulović, V.; Snaith, H. J.; Ginger, D. S.; Stranks, S. D. Photo-induced halide redistribution in organicinorganic perovskite films. Nat. Commun. 2016, 7, 11683.

(29) Futscher, M. H.; Lee, J. M.; McGovern, L.; Muscarella, L. A.; Wang, T.; Haider, M. I.; Fakharuddin, A.; Schmidt-Mende, L.; Ehrler, B. Quantification of ion migration in $\mathrm{CH}_{3} \mathrm{NH}_{3} \mathrm{PbI}_{3}$ perovskite solar cells by transient capacitance measurements. Mat. Horiz. 2019, 6, 1497--1503.

(30) Karmonik, C.; Hempelmann, R.; Cook, J.; Guthoff, F. Investigation of the proton migration mechanism in the perovskite proton conductor $\mathrm{BaCa}_{1.18} \mathrm{Nb}_{1.82} \mathrm{H}_{0.2} \mathrm{O}_{8.83}$ by means of quasielastic neutron scattering. Ionics 1996, 2, 69-74.

(31) Park, B. w.; Seok, S. I. Intrinsic Instability of InorganicOrganic Hybrid Halide Perovskite Materials. Adv. Mater. 2019, 31.

(32) Cardenas-Daw, C.; Simon, T.; Stolarczyk, J. K.; Feldmann, J. Migration of Constituent Protons in Hybrid Organic-Inorganic Perovskite Triggers Intrinsic Doping. J. Am. Chem. Soc. 2017, 139, 16462-16465.

(33) Sadhu, S.; Aqueche, K.; Buffeteau, T.; Vincent, J. M.; Hirsch, L.; Bassani, D. M. Unexpected surface interactions between fluorocarbons and hybrid organic inorganic perovskites evidenced by PM-IRRAS and their application towards tuning the surface potential. Mat. Horiz. 2019, 6, 192-197.

(34) Ramin, M. A.; Le Bourdon, G.; Daugey, N.; Bennetau, B.; Vellutini, L.; Buffeteau, T. PM-IRRAS Investigation of SelfAssembled Monolayers Grafted onto $\mathrm{SiO}_{2} / \mathrm{Au}$ Substrates. Langmuir 2011, 27, 6076-6084.

(35) Méndez-Ardoy, A.; Markandeya, N.; Li, X.; Tsai, Y.-T.; Pecastaings, G.; Buffeteau, T.; Maurizot, V.; Muccioli, L.; Castet, F.; Huc, I.; Bassani, D. M. Multi-dimensional charge transport in supramolecular helical foldamer assemblies. Chem. Sci. 2017, 8, $7251-7257$.

(36) Liang, C.-K.; Dubacheva, G. V.; Buffeteau, T.; Cavagnat, D.; Hapiot, P.; Fabre, B.; Tucker, J. H. R.; Bassani, D. M. Reversible Control over Molecular Recognition in Surface-Bound Photoswitchable Hydrogen-Bonding Receptors: Towards ReadWrite-Erase Molecular Printboards. Chem. Eur. J. 2013, 19, 1274812758.

(37) Buffeteau, T.; Desbat, B.; Turlet, J. M. Polarization Modulation FT-IR Spectroscopy of Surfaces and Ultra-Thin Films: Experimental Procedure and Quantitative Analysis. Appl. Spectrosc. 1991, 45, 380-389.

(38) A small amount of exchange takes place during the setup of the experiment but does not affect measurements.

(39) Crank, J. The Mathematics of Diffusion Oxford University Press: Oxford, 1975.

(40) The difference in reactivity between the two intermediate species can result from the combined effects of statistics and kinetic isotope effects.

(41) Khawam, A.; Flanagan, D. R. Basics and applications of solid-state kinetics: A pharmaceutical perspective. J. Pharm. Sci. 2006, 95, 472-498

(42) Grunwald, E.; Ku, A. Y. Proton exchange between ammonium ion, trimethyl-ammonium ion, and water. Speed of the dehydration step that precedes bimolecular proton transfer. $J$. Am. Chem. Soc. 1968, 90, 29-31.

(43) We observe that the location of the maximum rate as a function of incident radiation power shifts to higher values $(1 \mathrm{~mW})$ for 
thinner 80-nm samples, presumably due interfacial trapping of the charge carriers.

(44) Kim, G. Y.; Senocrate, A.; Yang, T.-Y.; Gregori, G.; Grätzel, M.; Maier, J. Large tunable photoeffect on ion conduction in halide perovskites and implications for photodecomposition. Nat. Mater. 2018, 17, 445-449.

(45) Bag, M.; Renna, L. A.; Adhikari, R. Y.; Karak, S.; Liu, F.; Lahti, P. M.; Russell, T. P.; Tuominen, M. T.; Venkataraman, D. Kinetics of Ion Transport in Perovskite Active Layers and Its Implications for Active Layer Stability. J. Am. Chem. Soc. 2015, 137, 13130-13137.

(46) Senocrate, A.; Moudrakovski, I.; Acartuerk, T.; Merkle, R.; Kim, G. Y.; Starke, U.; Graetzel, M.; Maier, J. Slow $\mathrm{CH}_{3} \mathrm{NH}_{3}{ }^{+}$ Diffusion in $\mathrm{CH}_{3} \mathrm{NH}_{3} \mathrm{PbI}_{3}$ under Light Measured by Solid-State NMR and Tracer Diffusion. J. Phys. Chem. C 2018, 122, 21803-21806.

(47) We calculate that for the 80-nm thick sample, $97.5 \%$ of incident photons are absorbed. De Wolf, S.; Holovsky, J.; Moon, S.J.; Löper, P.; Niesen, B.; Ledinsky, M.; Haug, F.-J.; Yum, J.-H.; Ballif, C. Organometallic Halide Perovskites: Sharp Optical Absorption Edge and Its Relation to Photovoltaic Performance. $J$. Phys. Chem. Lett. 2014, 5, 1035-1039.
(48) Müller, C.; Glaser, T.; Plogmeyer, M.; Sendner, M.; Döring, S.; Bakulin, A. A.; Brzuska, C.; Scheer, R.; Pshenichnikov, M. S.; Kowalsky, W.; Pucci, A.; Lovrinčić, R. Water Infiltration in Methylammonium Lead Iodide Perovskite: Fast and Inconspicuous. Chem. Mater. 2015, 27, 7835-7841.

(49) The calculated 1D diffusion constant increases to $2.1 \pm 0.4 \mathrm{x}$ $10^{-14} \mathrm{~cm}^{2} \mathrm{~s}^{-1}$ for the same 350 -nm thick sample under illumination at $0.5 \mathrm{~mW} \mathrm{~cm}{ }^{-2}$ irradiance. The 1D diffusion constants are estimated using the approximation $k=\left(\pi^{2} / 4 L^{2}\right) D$, where $L$ is the sample thickness. See ref. (39).

(50) Egger, D. A.; Kronik, L.; Rappe, A. M. Theory of Hydrogen Migration in Organic-Inorganic Halide Perovskites. Angew. Chem. Int. Ed. 2015, 54, 12437-12441.

(51) Bourgoin, J. C.; Corbett, J. W. A new mechanism for interstistitial migration. Phys. Lett. A 1972, 38, 135-137.

(52) Song, Z.; Wang, C.; Phillips, A. B.; Grice, C. R.; Zhao, D.; Yu, Y.; Chen, C.; Li, C.; Yin, X.; Ellingson, R. J.; Heben, M. J.; Yan, Y. Probing the origins of photodegradation in organic-inorganic metal halide perovskites with time-resolved mass spectrometry. Sustain. Energ. Fuels 2018, 2, 2460-2467. 


$$
\rho_{-}^{\circ}
$$

